\title{
Competencia transversal pensamiento crítico: Su caracterización en estudiantes de una secundaria de México
}

\author{
Crosscurricular Competency of Critical Thinking: \\ Its Characterization in Students of One Mexico High School
}

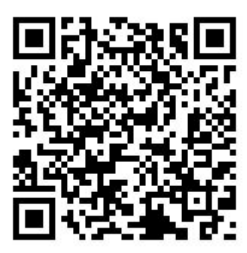

\author{
Candelaria Molina-Patlán \\ Tecnológico de Monterrey \\ Monterrey, México \\ candelaria.molina.p@hotmail.com \\ Gloria Perla Morales-Martínez² \\ Tecnológico de Monterrey \\ Monterrey, México \\ gpmoralesm@gmail.com \\ Jaime Ricardo Valenzuela-González ${ }^{3}$ \\ Tecnológico de Monterrey \\ Monterrey, México \\ jrvg@itesm.mx
}

Recibido 24 de noviembre de 2014 • Corregido 6 de noviembre de 2015 • Aceptado 17 de noviembre de 2015

\footnotetext{
${ }^{1}$ Realizó estudios profesionales en Licenciatura en Educación Primaria en la Escuela Normal "Miguel F. Martínez" y en Licenciatura en Educación Media. Especialidad en Matemáticas en la Escuela Normal Superior "Moisés Sáenz Garza". Actualmente cursa la Maestría en Administración de Instituciones Educativas con acentuación en Educación Básica en el Instituto Tecnológico de Monterrey. Su experiencia de trabajo ha girado, principalmente, alrededor del campo educativo, específicamente en el área de educación primaria desde hace once años. En la actualidad Candelaria Molina Patlán funge como docente frente a grupo en la Escuela Primaria "Profr. David Colunga Galicia" atendiendo a un grupo de segundo grado.

${ }^{2}$ Es Ingeniera Bioquímica por el ITESM. Cuenta con Maestría en Administración de Instituciones Educativas y otra en Asesoramiento Educativo Familiar, la primera por el ITESM, y la segunda, por el Centro Universitario Villanueva de España. Labora en el Liceo de Monterrey como Directora Administrativa. Sus investigaciones son en el campo de la transferencia del aprendizaje y como colaboradora y asesora en temas de evaluación institucional.

${ }^{3}$ Doctor en Psicología Educativa por la Universidad de Texas en Austin. Profesor-investigador en la Escuela de Graduados en Educación del Tecnológico de Monterrey. Áreas de investigación: evaluación educativa, educación a distancia y educación basada en competencias. Miembro del Sistema Nacional de Investigadores del CONACYT. Coautor de Fundamentos de investigación educativa (tres volúmenes, en formato de eBook) publicado por la Editorial Digital Tecnológico de Monterrey.
} 
doi: http://dx.doi.org/10.15359/ree.20-1.11

URL: http://www.una.ac.cr/educare

CORREO: educare@una.cr

Resumen. El presente artículo deriva de un trabajo de investigación cuyo objetivo fue determinar las características del estudiantado de secundaria considerado con dominio de la competencia transversal desarrollo del pensamiento crítico. El estudio tuvo un enfoque post positivista. Empleó la metodología mixta en su modalidad de secuencial exploratoria. En la fase cualitativa se recolectaron datos aplicando entrevistas semiestructuradas a seis estudiantes de primero y segundo de secundaria; los resultados fueron analizados mediante una codificación inductiva. En la fase cuantitativa se aplicó un autorreporte con escala Likert a 50 estudiantes de secundaria. Los datos de esta etapa se sometieron a análisis descriptivo, psicométrico y de relaciones. Se descubrió que el alumnado de secundaria competente en desarrollo de pensamiento crítico tiene habilidades de resolución de problemas, de emisión de juicios y de disposición hacia el pensamiento crítico. Se destaca su solidaridad, el gusto por compartir su conocimiento y por ayudar a quien se lo solicita. También se encontró que es mínima la diferencia de esta competencia entre hombres y mujeres, aunque en el contexto del aula se reflejen más las capacidades de las mujeres. Estos hallazgos pueden ser tomados en cuenta en la práctica educativa para impulsar el desarrollo del pensamiento crítico en estudiantes $y$, a la vez, su aprendizaje; puesto que al fortalecer la competencia del pensamiento crítico mediante la práctica, se ponen en juego diferentes habilidades, conocimientos y actitudes, lo cual es afín al enfoque de educación actual en México, al enfoque basado en competencias.

Palabras claves. Competencia, competencia transversal, pensamiento crítico.

Abstract. This article is derived from a research study whose objective was to determine the characteristics that high school students have which are considered to be competent in the crosscurricular competence development of critical thinking. The study had a post positivist approach. It used mixed methodology in sequential exploratory mode. In the qualitative phase, data was collected by applying semi-structured interviews to six students of the first and second high school years; the results were analyzed using an inductive encoding. In the quantitative phase a self-report with Likert scale was applied to 50 high school students. The data, at this stage, were submitted to descriptive, psychometric and relations analysis. It was found that high school students, competent in developing critical thinking, feature problem-solving, issuance of judgments and disposition towards critical thinking skills. Their solidarity, the taste for sharing their knowledge and for helping those who prompted them were highlighted. We also found that the difference between men and women in this competition is minimal, but in the classroom context the capacity of women is reflected more. These findings can be taken into account in the educational practice to boost the development of critical thinking in students, and at the same time, their learning, because knowledge, attitudes and different skills come into play when strengthening critical thinking competency through practice, which is akin to the current educational approach in Mexico, to the skills-based approach.

Keywords. Competition, crosscurricular competency, critical thinking.

El mundo está inmerso en la sociedad del conocimiento y de la Información, en la cual, por los avances tecnológicos y la diversidad de medios de comunicación existentes, se puede tener acceso a un sinfín de información y oportunidades. Esta condición demanda que los individuos estén mejor preparados para comprender e interactuar en el complejo contexto que les toca vivir. 
Por ello, no es suficiente que las personas cuenten con conocimientos y capacidades, sino que han de ser formadas de manera que se fomenten, movilicen y apliquen diversas capacidades y actitudes. La misión de la escuela, en este caso la escuela secundaria, ya no es llenar de conocimientos al estudiantado, sino desarrollarles en diferentes competencias para la vida. Involucra aprender a aprender, aprender a hacer, aprender a convivir y aprender a ser con el fin de contribuir al desarrollo cognitivo y personal del alumnado (Delors, 1997) de forma que pueda desempeñarse eficientemente en los diferentes entornos en los cuales se desenvuelva.

Basándose en esta premisa, entonces es oportuno investigar sobre las competencias, pero sobre todo en aquellas que son aplicables a la vez a diferentes disciplinas. Estas son las denominadas competencias genéricas o transversales que, por su naturaleza multidisciplinaria ofrecen más oportunidades de aplicación y de aprendizajes.

Una de las principales competencias transversales que la Organización para la Cooperación y el Desarrollo Económico (OCDE, 2005, 2010) y la Unesco (2005) señalan como necesaria para poder desempeñarse eficientemente en la sociedad de la información y la comunicación, es el desarrollo del pensamiento crítico. Esto, debido a que es una habilidad de orden superior que requiere cuestionar, indagar, analizar, valorar, reflexionar, argumentar y juzgar sobre la información o situación que se presente.

Montoya (2007) menciona que hay muchas investigaciones que resaltan el hecho de que la mayor parte de los países tienen el problema de la carencia de un pensamiento crítico en sus estudiantes. Señala en especial a estudiantes de educación secundaria, donde es frecuente que los maestros y maestras se quejen de ello al externar frases como "los alumnos no analizan, solo reciben y no cuestionan información, no piensan, saben pero no aplican ni trasladan su conocimiento".

Además, se destaca el hecho de que el estudiantado que ingresa a la universidad no tiene las bases idóneas sobre esta competencia. Esta barrera impide que actúe eficientemente en sus clases y logren obtener una buena calidad en sus aprendizajes para poder ser profesionistas competentes (Madariaga y Schaffernicht, 2013).

Por ello, es importante profundizar sobre la competencia desarrollo de pensamiento crítico, sobre todo en el nivel de secundaria que es base para el estudiantado de educación superior que en un futuro serán la fuerza laboral del país.

Con el propósito de aportar y difundir conocimiento sobre el tema, el presente estudio se enfoca hacia la caracterización de personas (estudiantes) que se destacan en el uso de la competencia transversal denominada desarrollo del pensamiento crítico. Esta, como lo mencionan diversos autores (Beltrán y Torres, 2009; Facione, 2007; Halpern, 1998; Nieto y Saíz, 2011; OCDE, 2005, 2010), implica en el individuo cuestionar, razonar y reflexionar lo que 
doi: http://dx.doi.org/10.15359/ree.20-1.11

URL: http://www.una.ac.cr/educare

CORREO: educare@una.cr

se le presenta, ya sea información, un problema o cualquier situación de su realidad, incluso analizarse a sí mismo; todo con el fin de dar solución a una problemática o emitir un juicio. Además, permite al individuo transformarse, reestructurar su ser y, en consecuencia, valorar y modificar su entorno (Montoya, 2007).

\section{Definición del problema}

La competencia de un individuo se hace presente en la acción sobre alguna situación, al integrar los saberes, habilidades y actitudes que se poseen para dar solución a dicha situación. El desarrollo del pensamiento crítico es una de las principales competencias puesto que de manera juiciosa y reflexiva permite a la persona discriminar la información y decidir cómo actuar en consecuencia (Ennis, 1993, 1996).

Se han realizado diversos estudios sobre el pensamiento crítico, su aplicación o técnicas para desarrollarlo; pero poco se ha investigado sobre las características de las personas competentes en este tema y menos aún en el nivel de secundaria. Explorar sobre este enfoque para hacer aportaciones es lo que se pretendió con este trabajo.

El objetivo general que guio el estudio fue determinar los rasgos característicos de alumnos y alumnas de secundaria que se consideran competentes en la competencia transversal desarrollo del pensamiento crítico. Así, también se buscó identificar si sus atributos coincidían con los que se señalan en la bibliografía, describir la manera en que hacen evidente su competencia en desarrollo de pensamiento crítico y determinar si existen diferencias entre competentes en desarrollo de pensamiento crítico del género masculino y del femenino.

\section{Delimitación del estudio}

La investigación se desarrolló en el nivel educativo de educación básica (que abarca desde educación preescolar hasta educación secundaria, en un rango de edades de 3 a 15 años de edad), específicamente en una escuela secundaria de índole general, perteneciente al sistema federal y al sector público, ubicada al norte de la ciudad de Monterrey, Nuevo León, México. Participaron 50 estudiantes de primer grado de secundaria y tres de segundo grado de secundaria.

Se decidió trabajar con este nivel escolar porque la formación de estos alumnos y alumnas es fundamental para que puedan continuar con su aprendizaje en los siguientes niveles educativos. Tres estudiantes de segundo grado de secundaria (de 14 años de edad) se eligieron por la información que pueden aportar a la investigación al tener un poco más de experiencia y experticia en la competencia que se estudia. 
El desarrollo del pensamiento crítico coadyuva a lograr los rasgos del perfil de egreso del alumnado de educación básica de México. Esto se observa en el plan de estudios vigente que plantea competencias que incluyen argumentar y razonar situaciones, analizar, emitir juicios, interpretar y tomar decisiones, manejar eficientemente la información, interpretar y explicar diferentes procesos, actuar con responsabilidad, etc. (SEP, 2011).

El desarrollo de esta capacidad constituye un área de oportunidad de la educación actual. Esta competencia requiere reforzarse en las escuelas, pero sobre todo en el nivel de secundaria por el impacto que tiene en la educación superior. Una buena contribución para poder lograr avances es conocer las características de quienes piensan críticamente para, con base en ello, diseñar y realizar mejores prácticas educativas.

\section{Antecedentes prácticos}

De acuerdo con los diferentes estudios consultados sobre el desarrollo del pensamiento crítico desarrollados en el contexto mexicano y colombiano (Díaz-Barriga, 2001; Duran y Şendag, 2012; Guzmán y Sánchez, 2006; Olivares y Heredia, 2012; Ruiz, 2013), se determinó que el estudiantado tiene capacidades que no saben cómo emplear o potenciar. Para mejorarlas requiere que se les imparta una enseñanza tal que le permita movilizar sus conocimientos y capacidades, que confronte sus saberes, que relacione eventos y que promueva más reflexión.

Se comprobó que el empleo de estrategias o programas como el aprendizaje basado en problemas o el trabajo por proyectos durante el trabajo de aula permite al alumnado un mayor desarrollo de las habilidades de pensamiento crítico. Entre ellas, la reflexión, la inducción, la deducción, la inferencia, el análisis, la construcción de explicaciones, de juicios críticos y la toma de decisiones.

También se descubrió queese tipo de estrategiasfavorecela comunicación, la participación, la colaboración, la interacción y el desenvolvimiento del estudiante en la escuela y en otros ámbitos (Montoya y Monsalve, 2008).

Un descubrimiento muy importante es que para un mejor desempeño de las habilidades del pensamiento crítico, se requiere de la disposición y la motivación hacia la utilización de las competencias que se tienen, puesto que sin estas disposiciones, las habilidades de pensamiento crítico carecen de sentido (Escurra y Delgado, 2008; Giancarlo, Blohom y Urdan, 2004).

Es preciso indicar que la formación docente debe tomar en cuenta la capacitación de docentes en el conocimiento y la práctica de estrategias que ayuden a desarrollar el pensamiento crítico de sus estudiantes para que las puedan implementar en el desarrollo de sus clases.

En relación con las investigaciones previas, se tiene que hay muchas que abordan el tema del pensamiento crítico, pero desde un enfoque de diseño de estrategias de enseñanza o de 
doi: http://dx.doi.org/10.15359/ree.20-1.11

URL: http://www.una.ac.cr/educare

CORREO: educare@una.cr

evaluación de las habilidades y disposiciones hacia este. Dichas exploraciones se han realizado mayormente en el nivel de estudios superior y falta información sobre lo que se puede dar en la educación básica. Por tanto, los resultados presentados en este estudio son una aportación más para que se puedan buscar o diseñar acciones que favorezcan el desarrollo del pensamiento crítico desde edades tempranas, desde la educación básica, lo cual ayudaría a la mejora de la calidad educativa y a la transformación de la sociedad. Cabe señalar que la mayoría de los estudios mencionados se realizaron en el contexto mexicano.

\section{Marco teórico}

\section{Concepto de competencia}

La competencia es"una manifestación transversal de los componentes actitudinal, técnico, procedimental y social" (García-San Pedro, 2009, p. 15). Ayuda a las personas a comprender una situación, a ser efectivas y eficientes, a tomar conciencia, a ser críticas, a afrontar retos, a asumir la responsabilidad de los actos y a regirse por indicadores de calidad (Fernández-Salinero, 2006; Tobón, 2004, citados por García-San Pedro, 2009).

La competencia representa la capacidad de afrontar demandas complejas en un contexto determinado.... se construye en cuanto integración de habilidades cognitivas y prácticas asociadas, saberes, creatividad, motivación, valores, actitudes, emociones y otros componentes sociales y de la conducta que se movilizan para efectuar una acción efectiva. (Sobrado y Fernández, 2010, p. 18)

De acuerdo con estas disertaciones, la competencia va más allá de un simple uso de saberes y destrezas. Implica también aspectos sociales y morales para actuar de manera responsable y efectiva en una situación dada. Esa combinación da facultad para actuar de manera asertiva, consciente, responsable y eficiente ante una situación determinada que requiere una toma de decisiones o una solución. Además, permite plantear problemas, extrapolar y reajustar los saberes (Perrenoud, 2008).

García-San Pedro (2009) señala que las competencias pueden ser de varios tipos: básicas, genéricas específicas y meta-competencias. De ellas sobresalen las competencias genéricas o transversales por la implicación que tienen en diferentes ámbitos, y por su flexibilidad para manifestarse y para contribuir en el contexto de diferentes disciplinas, ya que permiten relacionarlas y adaptarlas de manera que se obtenga el máximo provecho.

En los últimos años en el terreno educativo más que competencias transversales, se ha manejado mucho la transversalidad con base en dos líneas de interés o enfoques. Primeramente se ha utilizado la transversalidad para vincular asignaturas con el fin de mejorar la comprensión 
de estas mismas y facilitar su aprendizaje. La segunda línea de interés es abordar problemáticas sociales como temas transversales dentro del currículo para concientizar sobre ellos al alumnado y formarlo de manera que se sensibilice con estos fenómenos sociales y colabore en su solución y erradicación para constituir un mundo mejor.

Sin menospreciar esos enfoques, es preciso dar mayor atención a las competencias transversales porque permiten al individuo desempeñarse en diferentes disciplinas, ámbitos o contextos a lo largo de toda la vida.

Trasladando esa idea al ámbito escolar, las competencias transversales pueden favorecer en el alumnado un mejor aprendizaje al intervenir en varias áreas del conocimiento, facilitando la apropiación de aprendizajes y el desarrollo de capacidades.

El plan de estudios del sistema educativo mexicano (SEP, 2011) determina las competencias para la vida, el perfil de egreso estudiantil de educación básica así como los estándares curriculares y los aprendizajes esperados que a su vez marcan el nivel de logro del desempeño del alumnado. Estos aspectos conforman el trayecto formativo que está organizado en cuatro campos de formación: lenguaje y comunicación, pensamiento matemático, exploración y comprensión del mundo natural y social, y el desarrollo personal y para la convivencia, cuyos contenidos son abordados en todos los grados de preescolar, primaria y secundaria, pero de manera gradual.

Otras características del plan de estudios de educación básica en México son que pretende el desarrollo de competencias, centra su atención en el alumnado, busca desarrollar al máximo sus aprendizajes a través de retos intelectuales. Estipula la creación de ambientes de aprendizaje de comunicación, interacción y colaboración, así como el uso de diferentes materiales escritos, manipulables, audiovisuales, multimedia, etc. La evaluación tiene la finalidad de mejorar el aprendizaje y la práctica docente.

Existe una gran variedad de competencias genéricas o transversales que pueden favorecer el aprendizaje. Algunas de ellas son el aprender a aprender, la resolución de problemas, el manejo de las tecnologías de la comunicación y de la información, investigar, comunicar, trabajar colaborativamente y desarrollar el pensamiento crítico.

\section{Competencia desarrollo del pensamiento crítico}

Definición de pensamiento crítico. Para comprender mejor la competencia desarrollo de pensamiento crítico es propicio considerar el significado del pensamiento en general.

El pensamiento es una facultad mental en la cual se reflexionan, cuestionan, examinan y manipulan las estructuras del conocimiento. La finalidad es dar una solución o emitir un juicio sobre algún asunto que se desea resolver, conocer o entender (Real Academia Española, 2001; McShane, Gillis y Benton, 2013). 
doi: http://dx.doi.org/10.15359/ree.20-1.11

URL: http://www.una.ac.cr/educare

CORREO: educare@una.cr

Paul y Elder (2003) mencionan que todas las personas piensan, pues es parte de su naturaleza. No obstante, gran parte de los pensamientos son arbitrarios, desinformados, parciales y con prejuicios. Por tanto, se debe trabajar sobre ello dado que del grado de refinamiento de los pensamientos depende la calidad de lo que elaboren o construyan las personas. De ahí la importancia de adiestrar el pensamiento para llevarlo a un orden superior como es el caso del pensamiento crítico.

Dewey (1998) señala que el pensamiento es automático y que está determinado por una serie de ideas e imágenes que transitan por la mente sin ninguna regulación. También expone que existe otro tipo de pensamiento superior al que denomina pensamiento reflexivo, el cual consiste en un encadenamiento de ideas que tienen una finalidad o meta a la cual llegan después de ser probadas mediante un análisis y una fundamentación.

El pensamiento reflexivo es equiparable al pensamiento crítico, es razonado, orienta o guía la acción (Ennis, 1993, 1996). El pensamiento crítico conlleva autorregulación y buen juicio (Facione, 1990; Lipman, 1988), por tanto, es un proceso de mayor discernimiento y conciencia, implica conocer el origen y razón de su conocimiento para poder utilizarlo (Kuhn, 1999).

Por otro lado, Paul y Elder (2005) consideran que el pensamiento crítico es un proceso en el que se analiza y evalúa la calidad del pensamiento con la finalidad de mejorarlo, asimilando las estructuras o elementos del pensamiento. Esto solo se logra mediante el uso de los estándares intelectuales universales como: el criterio, la claridad, la precisión, la relevancia, la profundidad, la amplitud, la lógica, la importancia y la justicia.

Una definición muy completa es la emitida por la Asociación Filosófica Americana (1990, citado por Facione, 2007, p. 21) "El pensamiento crítico (PC) es el juicio auto regulado y con propósito que da como resultado interpretación, análisis, evaluación e inferencia, como también la explicación de las consideraciones de evidencia, conceptuales, metodológicas, criteriológicas o contextuales en las cuales se basa ese juicio".

Tomando en cuenta diferentes estudios (Beltrán y Torres, 2009; Halpern, 1998; Montoya y Monsalve, 2008; Nieto y Saiz, 2011) se concluye que el pensamiento crítico es un pensamiento de orden superior, analítico, reflexivo y liberador. Implica cuestionar y manejar habilidades intelectuales para usar adecuadamente los conocimientos que se tienen y valorar las opiniones de las demás personas. Esto se hace con el propósito de autorregular el pensamiento, emitir juicios, tomar las mejores decisiones para responder a situaciones problemáticas, apreciar y transformar la realidad y crecer en desarrollo personal.

Factores del pensamiento crítico. Diversos autores (Facione, 1990, 2007; Nieto y Saiz, 2011; Tishman, Jay y Perkins, 1993) consideran que el pensamiento crítico requiere de la conjunción de varios factores para que se pueda desarrollar: habilidades de pensamiento, habilidades disposicionales y comprensión epistemológica del pensamiento crítico. 
Las habilidades del pensamiento crítico son aquellas que llevan una carga cognitiva, que requieren el uso de las estructuras mentales. Entre ellas están el conocimiento, la interpretación, el análisis, la inferencia, la explicación, la evaluación, la autorregulación, la meta cognición (Facione, 1990, 2007; López, 2012), el razonamiento verbal, habilidades investigativas, la probabilidad e incertidumbre, la toma de decisiones y la solución de problemas (Halpern, 1998).

La base de las habilidades del pensamiento crítico consiste en que la persona tenga conciencia de lo que conoce, de cómo lo sabe, así como del uso eficaz de sus recursos cognitivos (Kuhn, 1999).

En cuanto a la disposición, se habla del espíritu crítico que radica en la curiosidad por buscar la razón y el deseo de tener más información confiable y la disposición de apertura de ideas y de espíritu aventurero; orientación al aprendizaje y al dominio; afán por aclarar e intentar saber, planificar, y tener estrategias y apertura mental; búsqueda y evaluación de razones; la solución creativa e integridad cognitiva (Giancarlo et al., 2004; Tishman et al., 1993).

Diversos estudios (Dewey, 1998; Ennis, 1993, 1996; Escurra y Delgado, 2008; Facione, 2007) refieren que para desarrollar el pensamiento crítico se debe tener mente abierta, estar en búsqueda constante de información y ser responsable de las consecuencias de las decisiones. Estos aspectos personales junto con el uso eficaz de las habilidades cognitivas conducen a mejores tomas de decisiones y juicios en una situación dada. Sears y Parsons (1991, citados por Halpern, 1998) concretan estas ideas al indicar que la actitud de identificar el momento en que es necesario aplicar una habilidad, y tener el deseo y la voluntad de hacerlo es lo que se denominaría ética de una persona pensadora crítica.

Nieto y Saiz (2011) comentan que han surgido nuevos factores indispensables para llegar a un pensamiento crítico. Estos factores son la comprensión epistemológica del pensamiento crítico, tener un buen nivel de desarrollo epistemológico y de las creencias sobre el pensamiento.

Al respecto Bailin (1999) sostiene que la "epistemological understanding is ... the central concept through which critical thinking is conceptualized [comprensión epistemológica...es el concepto central a través del cual se conceptualiza el pensamiento crítico]" (p. 167). Argumenta que esta epistemología se logra en la práctica, al evaluar la naturaleza del pensamiento, entender su sentido, razonarlo, justificarlo y ser capaz de aplicarlo de acuerdo con el contexto que lo requiere.

Puesto que toda actividad de pensamiento se sitúa en un grupo de supuestos o creencias epistemológicas que lo fundamentan, solo en una práctica constante se puede llegar a un mejor nivel de comprensión y un mejor manejo de la aplicación del pensamiento crítico.

Kuhn (1999) afirma que cuando una persona llega a tener una comprensión amplia sobre su conocimiento y su saber, entonces llega a un conocimiento epistemológico que es vital para ser más asertivo en el uso de estos mismos, valorar los hechos y seguir avanzando en su aprendizaje. 
doi: http://dx.doi.org/10.15359/ree.20-1.11

URL: http://www.una.ac.cr/educare

CORREO: educare@una.cr

En resumen, el pensamiento crítico requiere para su ejecución un conjunto de elementos como conocimiento, inferencia, evaluación, indagación, autorregulación, motivación, creatividad, dominio, eficiencia, apertura y meta cognición. La combinación e interrelación de todas estas habilidades, actitudes y tendencias favorecen su desarrollo.

Sin embargo, el pensamiento crítico se consolida mejor cuando se valora la naturaleza del pensamiento, su finalidad y su aplicación al contexto, llegando a obtenerse una comprensión epistemológica de este mismo.

Para quienes escriben este artículo, el pensamiento crítico es aquel pensamiento en el cual se ejecutan una serie de habilidades cognitivas y disposiciones con el fin de reflexionar sobre una situación para darle solución o emitir un juicio.

Proceso de desarrollo del pensamiento crítico. Como todo proceso, el desarrollo del pensamiento crítico se lleva a cabo en varias fases. Perkins (1992, citado por Cruz, 2010) establece tres etapas que conforman este proceso.

La primera etapa es prepararse para pensar. En ella se distinguen, por un lado, las motivaciones, intereses y creencias personales $y$, por otro, se toma conciencia de las habilidades o destrezas que se tienen hacia el pensamiento para poder actuar de manera eficaz y positiva. Esta es considerada una fase larga que requiere un cambio de actitud.

La segunda fase es la ejecución del pensamiento. En ella se requiere poner en juego el conjunto de habilidades cognitivas y meta cognitivas adecuadas para resolver un problema.

La tercera etapa se denomina como la comunicación del pensamiento. Consiste en retomar la meta cognición para controlar la información y el aprendizaje, de manera que se examine y comprenda qué, cómo, cuándo y con qué eficacia se aprende para luego ser capaz de comunicar dicho aprendizaje.

Como se puede apreciar en esta disertación de Perkins (1992, citado por Cruz, 2010), tanto las habilidades como las disposiciones del pensamiento crítico son importantes en el proceso de su desarrollo. A su vez, son complementarias e interactúan constantemente de tal forma que fortalecen la competencia.

\section{Características de una persona competente en desarrollo del pensamiento crítico}

López (2012,p.44) considera que el ser humano que maneja el pensamiento crítico"es aquel que es capaz de pensar por sí mismo". Un ser pensador crítico manifiesta seguridad de sí mismo, actúa con valores y busca siempre la verdad; pero sin considerarla absoluta. Así mismo, toma en cuenta las opiniones de otras personas, se orienta al cambio y tiene muchas disposiciones hacia 
el pensamiento como la curiosidad, mente abierta, auto confianza, orientación al futuro, ensayo y error (Brookfield, 1987, citado por Thomas, 2009; Facione, 2007; Paul y Elder, 2003, 2005).

Con estos rasgos es válido apuntar que esta persona se puede desempeñar de manera competente; pero, ¿se le podrá considerar como una persona experta? Para dar respuesta a esta pregunta se presenta a continuación la teoría de personas principiantes y expertas.

\section{Teoría de personas principiantes y expertas}

Se dice que tener conocimientos, habilidades y actitudes no es suficiente para tener competencia o experticia, entonces ¿qué se requiere para ser una persona experta?

En los últimos años lo que ha sido de mayor interés para entender el término competencia, es el estudio de tareas que requieran un conocimiento más complejo, el cual necesita de muchas horas de aprendizaje y de experiencia para obtenerse. Para entender y perfeccionar este nuevo enfoque, se han realizado estudios que detallan los contrastes entre las habilidades de las personas novatas y los expertas (Glaser, 1985).

Investigaciones acerca de resolución de problemas que impliquen un dominio cuantioso en conocimientos han dejado ver que existen sólidas interacciones entre las estructuras de conocimiento y los procesos del pensamiento.

Halpern (1998) menciona que cuantas más conexiones existen entre la información concentrada en la memoria, mayor es la posibilidad de recordarla. Es posible mejorar las estrategias de conocimiento, si se trabajan mejores maneras de exponer, de identificar y de usar las diferencias y particularidades del conocimiento (Glaser, 1985).

El estudio sobre individuos novatos/expertos en la resolución de problemas ha demostrado que hay relación entre las estructuras del conocimiento y los procesos cognitivos. Esta relación es medida con base en la calidad de las representaciones que el individuo realice de la situación o del problema. Tales representaciones se edifican sobre los conocimientos, su dominio y organización.

Glaser (1985) sostiene que las representaciones que un individuo novato puede hacer en referencia a un objeto o acontecimiento son solo una exposición literal y superficial del problema. Una persona novata con un poco más de capacidad llega a entender la situación, a tener conocimiento suficiente sobre ella, pero no sabe cómo aplicar ese conocimiento.

Por otra parte, las representaciones de una persona experta son más complejas, hacen inferencias sobre el problema, principios y abstracciones que se relacionan con este, organizan esas inferencias y son capaces de aplicar eficazmente los conocimientos (Glaser, 1985). 
doi: http://dx.doi.org/10.15359/ree.20-1.11

URL: http://www.una.ac.cr/educare

CORREO: educare@una.cr

Esas organizaciones de conocimientos de personas expertas son llamadas esquemas; constituyen la información y las estructuras de los conceptos acumulados en la memoria. Son prototipos que contienen lo que experimenta el sujeto, así como las interrelaciones del conocimiento de objetos, situaciones y sucesos que después son utilizados para percibir, interpretar, asimilar, inferir y construir conocimiento relacionado con esas estructuras internas (Glaser, 1985).

Glaser (1985) señala que los individuos expertos también tienen las capacidades de autorregulación o meta-cognición que los facultan para tomar conciencia de lo que saben y lo que no saben, para planificar con antelación a un suceso, hacer uso eficiente del tiempo y los recursos, así como para dar continuidad a la resolución de un problema. También comenta que generalizan sus competencias para poder utilizarlas hábilmente en diferentes áreas del conocimiento.

En esta disertación se aprecian características de desarrollo de pensamiento crítico, por lo que es muy probable que una persona experta sea una persona con esta competencia.

En conclusión, los individuos novatos no llegan a una comprensión total del problema, solo lo ven y tratan de manera superficial porque aún no tienen la capacidad de relacionar sus conocimientos con las situaciones que se les presentan. Los expertos, por el contrario, son muy perceptivos e inferenciales, logran una comprensión del problema, relacionan los hechos con los conocimientos y con sus experiencias. Además, reflexionan y conjugan todos sus recursos cognitivos y procedimentales para dar solución al problema.

Entonces, para la experticia, no basta con tener conocimientos, es necesario saber aplicarlos: utilizar más la reflexión, la abstracción, y enfrentar situaciones de reto. Las personas que desarrollan bien el pensamiento crítico pudieran considerarse expertas, puesto que los individuos expertos también argumentan, reflexionan, relacionan conocimientos, se guían por procesos y se basan en principios para explicar y comprender un fenómeno.

\section{Diferencia de pensamiento crítico debido al género}

En cuanto a este aspecto no hay mucha referencia de estudios. Sierra, Carpintero y Pérez (2010) descubrieron que no hay diferencias significativas en la competencia de pensamiento crítico entre alumnos y alumnas evaluadas en su estudio. 


\section{Método}

Se pretendió que esta investigación fuera una aproximación del paradigma pospositivista, ya que este es de naturaleza flexible y abierta, reconoce que el conocimiento es inacabado y que no se puede conocer totalmente la realidad. En línea con el paradigma pospositivista, se decidió utilizar una metodología de corte mixto. El procedimiento consistió en combinar la utilización de instrumentos de recolección de datos, análisis e interpretación tanto de métodos cualitativos como cuantitativos en el mismo estudio (Valenzuela y Flores, 2012). En esta aproximación fue útil la utilización del método de estudio de casos.

El método de estudio de casos permite una aproximación mixta ya que este instrumento radica en "una descripción y análisis a profundidad de un sistema delimitado" (Valenzuela y Flores, 2012, p. 90), lo cual da margen para obtener mayor cantidad de información sobre el fenómeno de estudio.

La presente investigación mixta se realizó en la modalidad que Valenzuela y Flores (2012) denominan secuencial exploratoria y que consiste de dos etapas consecutivas de diferentes métodos. Primero, se aplicó una etapa cualitativa mediante una entrevista semiestructurada a seis estudiantes de secundaria a quienes sus compañeros y compañeras, y sus docentes consideran competentes en desarrollo de pensamiento crítico. Posteriormente, en la segunda etapa se llevó a cabo la parte cuantitativa del estudio donde se recolectaron los datos mediante la aplicación de un test con escala Likert que midió tres dimensiones del pensamiento crítico: resolución de problemas, emisión de juicios y disposición hacia el pensamiento crítico.

\section{Etapa 1: Aproximación cualitativa}

Participantes. En esta etapa cualitativa colaboraron seis participantes, tres estudiantes de primer grado de secundaria y tres estudiantes de segundo grado de secundaria cuyas edades oscilan entre 12 y 14 años y a quienes sus compañeros y compañeras, y docentes consideran competentes en desarrollo de pensamiento crítico.

Instrumentos. Para esta etapa se aplicó la técnica de la entrevista semiestructurada dentro del propio contexto escolar de quienes participan. Las preguntas estuvieron relacionadas con la finalidad de que describieran cuáles características o cualidades tienen (Quecedo y Castaño, 2002) y perciben como propias en relación con la competencia de pensamiento crítico. En la tabla 1 se presenta cada pregunta y objetivo de investigación con las preguntas relacionadas que se incluyeron en las entrevistas realizadas. 
doi: http://dx.doi.org/10.15359/ree.20-1.11

URL: http://www.una.ac.cr/educare

CORREO: educare@una.cr

Tabla 1

Relación de las preguntas y objetivos de investigación con la entrevista semiestructurada

\begin{tabular}{|c|c|c|}
\hline Pregunta de investigación & Objetivo de investigación & Preguntas para entrevista \\
\hline $\begin{array}{l}\text { 1. ¿Cuáles características distinguen a } \\
\text { estudiantes de secundaria que destaquen } \\
\text { por su desarrollo de pensamiento crítico? }\end{array}$ & $\begin{array}{l}\text { 1. Determinar los atributos que caracterizan a } \\
\text { estudiantes competentes en desarrollo de } \\
\text { pensamiento crítico. }\end{array}$ & $\begin{array}{l}\text { ¿Te percibes a ti mismo(a) como competente en desarrollo } \\
\text { de pensamiento crítico? } \\
\text { ¿Cómo crees que desarrollaste tu competencia de } \\
\text { pensamiento crítico? } \\
\text { - ¿Cómo te describes en cuanto tu dominio en la } \\
\text { competencia de pensamiento crítico? ¿Qué sabes hacer y } \\
\text { qué no sabes hacer? }\end{array}$ \\
\hline $\begin{array}{l}\text { 2. ¿Cuáles son las coincidencias y diferencias } \\
\text { de las características encontradas en } \\
\text { estudiantes competentes en desarrollo de } \\
\text { pensamiento crítico con lo que señala la } \\
\text { bibliografía que fundamenta este estudio? }\end{array}$ & $\begin{array}{l}\text { 2. Identificar si los estudiantes competentes } \\
\text { en desarrollo de pensamiento crítico } \\
\text { muestran características específicas } \\
\text { que les diferencian de estudiantes no } \\
\text { competentes. } \\
\text { 3. Identificar y determinar si las } \\
\text { características detectadas en estudiantes } \\
\text { competentes en desarrollo de pensamiento } \\
\text { crítico coinciden con las que se señalan en } \\
\text { la bibliografía consultada. }\end{array}$ & $\begin{array}{l}\text { - ¿Cuáles cualidades tienes que ayudan a que uses mejor tu } \\
\text { pensamiento crítico? } \\
\text { · ¿Podrías dar algunos ejemplos que puedan ilustrar la } \\
\text { manera en que empleas tu pensamiento crítico? }\end{array}$ \\
\hline $\begin{array}{l}\text { 3. ¿Cómo se hace evidente la capacidad } \\
\text { de desarrollo de pensamiento crítico en } \\
\text { estudiantes de secundaria? }\end{array}$ & $\begin{array}{l}\text { 4. Descubrir la manera en que estudiantes de } \\
\text { secundaria hacen evidente su competencia } \\
\text { en desarrollo de pensamiento crítico. }\end{array}$ & $\begin{array}{l}\text { ¿Existen evidencias claras de un comportamiento } \\
\text { competente en desarrollo de pensamiento crítico en tus } \\
\text { actividades } 0 \text { acciones? ¿Cuáles serían esas evidencias? }\end{array}$ \\
\hline $\begin{array}{l}\text { 4. ¿Existe diferencias significativas entre } \\
\text { estudiantes competentes en desarrollo } \\
\text { de pensamiento crítico de acuerdo con } \\
\text { su género? }\end{array}$ & $\begin{array}{l}\text { 5. Determinar si existen diferencias entre } \\
\text { estudiantes competentes en desarrollo de } \\
\text { pensamiento crítico del género masculino } \\
\text { y del género femenino. }\end{array}$ & $\begin{array}{l}\text { ¿Qué te ha motivado a desarrollar la competencia de } \\
\text { pensamiento crítico? } \\
\text { - Con respecto a la competencia de pensamiento crítico, } \\
\text { ¿has notado algunas diferencias de ejecución en las } \\
\text { personas del otro sexo diferentes al tuyo? ¿Cuáles son? ¿A } \\
\text { qué crees que se deban? }\end{array}$ \\
\hline
\end{tabular}

Nota: Elaboración propia.

\section{Procedimientos}

Selección de la muestra. Para seleccionar a los sujetos participantes de esta etapa se realizó un sondeo aleatorio con el estudiantado y docentes de tres grupos de primer grado y tres grupos de segundo grado de secundaria. Se seleccionaron tres estudiantes de primer grado y tres estudiantes de segundo grado que tuvieron más menciones. Por conveniencia para la investigación, se procuró escoger tres hombres y tres mujeres. Una vez realizada la selección de participantes, se les informó el objetivo de la investigación, así como en qué consistiría su participación y también se les pidió su firma de autorización y la de sus padres o madres. La institución donde se realizó la investigación cuenta con seis grupos de cada grado escolar y por cuestiones de actividades 
internas se dio la oportunidad de realizar el sondeo solo en los grupos antes mencionados, esto no limitó la mención del estudiantado competente de los grupos que no fueron encuestados.

Aplicación del instrumento. En las entrevistas inicialmente se presentó la persona entrevistadora y platicó un poco con cada estudiante recordándole el objetivo de la investigación y lo valioso de su participación para obtener información sobre el tema. Durante el transcurso de la entrevista, se dio libertad al sujeto participante para hablar ampliamente y con soltura sobre el tema. Se agregaron algunas preguntas con base en las respuestas del estudiantado para así aclarar sus respuestas y obtener mayor información relevante sobre la competencia en desarrollo de pensamiento crítico (Valenzuela y Flores, 2012).

Es preciso aclarar que, con el permiso de los sujetos participantes, se grabó en audio todo el desarrollo de las entrevistas con el fin de transcribirlas en su totalidad para colectar todos los datos vertidos en ellas.

Estrategias de análisis. En este análisis se consideró el proceso de codificación inductivo propuesto por Creswell (2005, citado por Valenzuela y Flores 2012, p. 182), el cual contiene las siguientes fases: (1) Dar sentido a los datos, (2) dividir el texto en segmentos para separar ideas, (3) etiquetar los segmentos con códigos, (4) examinar los códigos en cuanto a traslape y redundancia, (5) segmentar esos códigos en temas más amplios.

\section{Etapa 2: Aproximación cuantitativa}

Participantes. En esta etapa de perfil cuantitativo se requirió la participación de 50 estudiantes de entre 12 y 14 años de edad que cursan el primer grado de secundaria ( 25 hombres y 25 mujeres). La muestra fue de tipo probabilística estratificada (Hernández, Fernández y Baptista, 2010), pues por conveniencia de una de las preguntas y objetivos de la investigación se requirió tener una cantidad equitativa de hombres y mujeres. Esta muestra se seleccionó aleatoriamente después de haber determinado los dos estratos de la población de estudiantes de primer año de secundaria.

Instrumentos. Mientras que el instrumento utilizado en la etapa cualitativa fue de tipo abierto y flexible, en esta etapa cuantitativa se hizo necesario implementar un instrumento de tipo cerrado para poder hacer una codificación objetiva y un análisis estadístico de los datos (Valenzuela y Flores, 2012). El tipo de test empleado fue un instrumento de autorreporte con escala Likert de uno a cuatro puntos, donde $1=$ casi nunca, $2=$ pocas veces, $3=$ algunas veces y $4=$ casi siempre.

En el test se presentó un conjunto de afirmaciones sobre tres dimensiones de pensamiento seleccionadas con base en el marco teórico que respalda al presente trabajo. Estas fueron la resolución de problemas, la emisión de juicios y las disposiciones hacia el pensamiento crítico. Para cada dimensión se elaboraron 10 ítems relacionados con algunas habilidades que se contemplan en las diferentes dimensiones. 
doi: http://dx.doi.org/10.15359/ree.20-1.11

URL: http://www.una.ac.cr/educare

CORREO: educare@una.cr

Todo instrumento de medición debe cubrir ciertos requisitos para ser capaz de generar información calificada. Estos requisitos son la confiabilidad y la validez (Hernández et al., 2010). Para determinar la confiabilidad de la consistencia interna del instrumento (que debe acercarse a 1.0) se utilizó el coeficiente alfa de Cronbach.

En cuanto a la validez, se cuidó que el test cubriera la validez de contenido al medir tres dimensiones de lo que es pensamiento crítico que estuvieran especificadas en la bibliografía y al diseñar los ítems de acuerdo con ella. Además se calcularon las correlaciones de Pearson de cada ítem con su escala respectiva para comprobar si los reactivos pertenecían a la escala en la cual fueron clasificados. Lo anterior también ayudó a cuidar la validez de constructo de la investigación.

Por otra parte, la validez de criterio se cuidó al complementar, comparar y contrastar los resultados cualitativos y cuantitativos obtenidos en las dos fases de la investigación.

\section{Procedimientos}

Selección de la muestra. La selección de 50 estudiantes participantes en esta etapa fue de forma aleatoria. Primeramente se pidieron a la dirección las listas de estudiantes de los seis grupos de primer grado. Con ellas se contabilizó el total de cada sexo para posteriormente dividir esa cantidad entre 25 y así determinar el rango adecuado para la selección de participantes. En esta misma visita a la institución se programó el día para la aplicación del instrumento.

Aplicación del instrumento. Una vez que se tuvieron las listas de 25 alumnos y 25 alumnas, con el apoyo de la dirección y por cuestiones de espacio, se determinó aplicar el autorreporte en dos partes. Se reunió a un primer grupo de participantes y se le explicó el objetivo de la investigación. Asimismo, se le invitó a colaborar informándole que su participación consistiría en contestar de forma anónima un test, cuya información sería totalmente confidencial y solo en relación con el tema del proyecto.

Se le proporcionó a cada estudiante una carta de consentimiento con la finalidad de recabar su firma y así quedó formalmente incluido como colaborador o colaboradora de este estudio. Acto seguido, se entregó el test a cada participantes y se le leyeron las instrucciones en general. Durante el proceso se aclararon las dudas al respecto y se subrayó la importancia de contestar honestamente para obtener resultados reales y confiables. Enseguida se le dio un tiempo de 60 minutos para que contestara el test y se estuvo vigilando para que todo se desarrollara de la mejor manera.

Estrategias de análisis de datos. Ya teniendo la totalidad de los datos colectados, se procedió a realizar el análisis.

a) Análisis descriptivo. En este análisis se determinó el grado de competencia de los alumnos y alumnas a nivel dimensión y a nivel global. Para ello se obtuvo la media aritmética, valores mínimos y máximos, rango, desviación estándar y coeficiente de asimetría. Con 
doi: http://dx.doi.org/10.15359/ree.20-1.11

URL: http://www.una.ac.cr/educare

CORREO: educare@una.cr

estos valores se dividió el total de participantes en cinco clases o agrupaciones de datos, los cuales permitieron, a su vez, determinar niveles de competencia.

- Clase A. No es competente.

- Clase B. Poco competente.

- Clase C. Regularmente competente.

- Clase D. Considerablemente competente.

- Clase E. Muy competente.

b) Análisis psicométrico. Con este análisis se evaluó la consistencia interna del instrumento mediante el cálculo del coeficiente alfa de Cronbach con el que se determinó que el instrumento en general tiene una fiabilidad elevada según Hernández et al. (2010). También se analizó la validez de contenido justificando las dimensiones manejadas en el instrumento con la teoría. Un tercer análisis psicométrico fue el de correlaciones, el cual se hizo con el coeficiente de correlación de Pearson, donde se vio la relación de pertenencia entre los reactivos de las diferentes dimensiones y entre dimensiones. Aquí los resultados fueron aceptables.

c) Análisis de relaciones. Para poder dar respuesta a una de las preguntas específicas de investigación, se estableció la comparación de promedios de las dimensiones y global de acuerdo con el género. Para ello se utilizó la herramienta tabla dinámica de Excel.

\section{Resultados, análisis y discusión}

Las características que distinguen al estudiantado competente en pensamiento crítico son resolución de problemas, emisión de juicio y disposiciones hacia el pensamiento crítico. Los resultadoscuantitativos refieren queel $42 \%$ delos 50 sujetos participantes sonconsiderablemente competentes o muy competentes $y$, además, un $32 \%$ se ubica en regularmente competente en pensamiento crítico (figura 1).

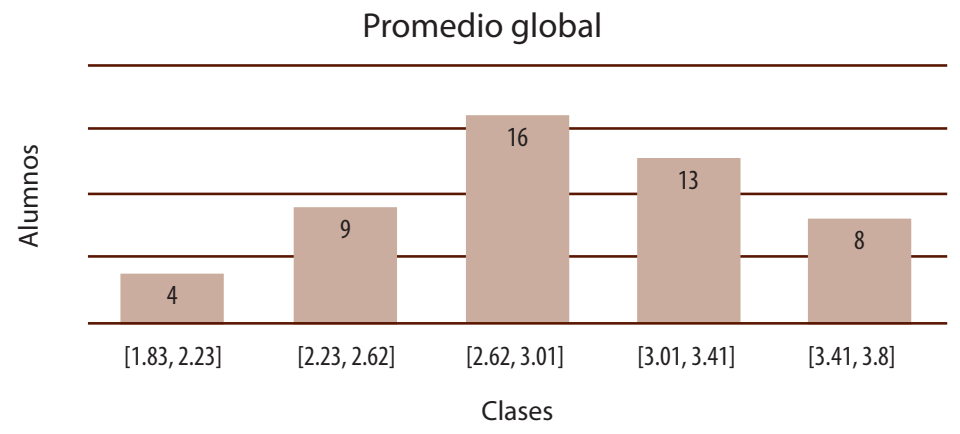

Figura 1. Histograma del promedio global. Las rangos corresponden a las clases A, B, C, D y E respectivamente. 
doi: http://dx.doi.org/10.15359/ree.20-1.11

URL: http://www.una.ac.cr/educare

CORREO: educare@una.cr

En congruencia con las dimensiones medidas en la fase cuantitativa, en el análisis cualitativo se destacaron en el estudiantado habilidades y actitudes enunciadas en la tabla 2.

Tabla 2

Características de personas competentes en pensamiento crítico

\begin{tabular}{|c|c|}
\hline Habilidades y actitudes & Cualidades específicas \\
\hline Dominio de ideas & $\begin{array}{l}\text { - Usan su conocimiento para resolver situaciones escolares y de la vida, mejorar } \\
\text { calificaciones, participar más en clase, dar respuesta a lo que les pregunten. } \\
\text { - Tienen habilidad lectora. Anticipan temas. }\end{array}$ \\
\hline Interpretar & - Usan la lógica. \\
\hline Analizar & $\begin{array}{l}\text { - Valoran las partes del problema o situación. } \\
\text { - Analizan y piensan antes de actuar. } \\
\text { - Usan estrategias de aprendizaje como esquemas, resúmenes, cuadros sinópticos, } \\
\text { problemas matemáticos. }\end{array}$ \\
\hline Inferir & $\begin{array}{l}\text { Elaboran conclusiones o resúmenes de la información que se les da para poder } \\
\text { resolver lo que se les pide. }\end{array}$ \\
\hline Reflexionar & $\begin{array}{l}\text { - Razonan mucho las cosas considerando cada argumento y desarrollo del tema. } \\
\text { - Comprenden mejor las cosas. } \\
\text { - Buscan la mejor alternativa de solución. }\end{array}$ \\
\hline Argumentar & $\begin{array}{l}\text { Saben explicar el tema. } \\
\text { - Expresan su opinión de acuerdo con lo que piensan y con lo que investigan. } \\
\text { - Defienden su postura. }\end{array}$ \\
\hline Autorregulación & $\begin{array}{l}\text { - Saben qué están pensando. } \\
\text { Piensan bien las cosas para evitar o corregir errores. }\end{array}$ \\
\hline Diversos puntos de vista & - Respetan el punto de vista de las demás personas. \\
\hline Orientación al aprendizaje & $\begin{array}{l}\text { - Desean saber más. } \\
\text { - Son personas curiosas. } \\
\text { - Leen por anticipado el tema de clase. } \\
\text { - Ansían desarrollar más su pensamiento. }\end{array}$ \\
\hline
\end{tabular}




\begin{tabular}{|c|c|}
\hline Habilidades y actitudes & Cualidades específicas \\
\hline \multirow{2}{*}{ Investigar } & Les gusta investigar. \\
\hline & - Investigan por iniciativa propia. \\
\hline \multirow{2}{*}{ Auto concepto } & Se consideran competentes en pensamiento crítico. \\
\hline & · Están conscientes de sus capacidades. \\
\hline \multirow{4}{*}{ Compartir conocimiento } & Dan a conocer a las demás personas lo que saben. \\
\hline & - Apoyan a sus compañeros y compañeras que tienen dudas. \\
\hline & - Explican a otras personas lo que entendieron, les ayudan a aprender. \\
\hline & - Dan ánimos a sus compañeros y compañeras que tienen problemas. \\
\hline \multirow{8}{*}{ Motivación } & - Tener buenas calificaciones. \\
\hline & - Demostrar que se pueden hacer mejor las cosas. \\
\hline & Ser alguien, superarse. \\
\hline & - Tener una carrera profesional. \\
\hline & - Mejorar económicamente. \\
\hline & - Ser ejemplo para las demás personas. \\
\hline & - Cuentan con el apoyo de sus familias. \\
\hline & - Desarrollar más su pensamiento crítico. \\
\hline
\end{tabular}

Nota: Elaboración propia con datos de etapa cualitativa.

Se coincide con la bibliografía en que el alumnado maneja tanto habilidades como disposiciones en el desarrollo de su pensamiento (Perkins, 1992, citado por Cruz, 2010). También hay correspondencia con el proceso de pensamiento nombrado por este mismo autor. El alumnado tiene motivaciones que lo llevan a actuar y desarrollar su pensamiento, hace uso de sus habilidades y conocimientos para resolver diversas situaciones y aprender de ellas. Después valora y reflexiona esos conocimientos para darlos a conocer al opinar o compartir sus saberes con las demás personas.

Las diferencias fueron que en las entrevistas los alumnos y alumnas resaltaron mucho el ser solidarios o solidarias con las demás personas y que su principal motivación es la superación personal. 
doi: http://dx.doi.org/10.15359/ree.20-1.11

URL: http://www.una.ac.cr/educare

CORREO: educare@una.cr

Por otro lado, en la bibliografía se da mucha relevancia a la autorregulación, meta-cognición o conocimiento epistemológico (Bailin, 1999; Facione, 1990, 2007; Kuhn, 1999; López, 2012), y esto no se encontró en los datos recolectados. Solo se identificó un poco de la autorregulación.

Otro aspecto no mencionado en las entrevistas es la creatividad, la cual es considerada por Giancarlo et al. (2004).

Los resultados mostraron que estudiantes de secundaria de Nuevo León, México resuelven problemas; afrontan situaciones; reflexionan; explican a otras personas lo que saben; se organizan; usan estrategias de aprendizaje como esquemas, resúmenes, cuadros sinópticos; investigan; analizan; dan sus propias opiniones; participan activamente en la clase y tienen buenas calificaciones. Estas características les permiten organizar, valorar y reflexionar sobre las situaciones para interactuar con su aprendizaje y obtener buenos resultados académicos que también pueden repercutir en su vida cotidiana.

El análisis de relaciones de la etapa cuantitativa arrojó como resultado que las mujeres tienen un poco más de habilidades y disposiciones que los hombres, aunque la diferencia no fue significativa: difieren entre 0.13 y 0.22 en los diversos promedios. Sin embargo, en las tres dimensiones medidas y en el promedio general, los valores de las mujeres están por arriba de la media aritmética (ver tabla 3).

Tabla 3

Comparación de promedios por escala, género y en relación con la media aritmética

\begin{tabular}{lcccc}
\hline Género & $\begin{array}{l}\text { Resolución de } \\
\text { problemas }\end{array}$ & Emisión de juicios & $\begin{array}{l}\text { Disposición hacia el } \\
\text { pensamiento }\end{array}$ & Promedio global \\
\hline Hombres & 2.71 & 2.85 & 2.91 & 2.82 \\
Media aritmética & $\mathbf{2 . 8 0}$ & $\mathbf{2 . 9 6}$ & $\mathbf{2 . 9 7}$ & $\mathbf{2 . 9 1}$ \\
Mujeres & 2.89 & 3.07 & 3.04 & 3.00 \\
\hline
\end{tabular}

Nota: Elaboración propia con datos de etapa cuantitativa.

En contraste, en los datos crudos de la etapa cualitativa tres participantes consideraron que tanto hombres como mujeres tienen las mismas capacidades para pensar, aunque a veces sus opiniones sean diferentes. Los otros tres participantes coincidieron en que las mujeres son más participativas, reflexivas y activas, y que tienen un pensamiento más desarrollado.

En referencia a los hombres opinan que son más toscos e impulsivos, que no piensan bien las cosas antes de actuar, que tienen cierto miedo a mostrar su inteligencia por temor a que sus congéneres se burlen de ellos, lo cual indica falta de seguridad y confianza en sí mismos. 


\section{Conclusiones}

Losalumnosy alumnas de secundaria de Nuevo León, México, competentes en desarrollo de pensamiento crítico tienen, por una parte, habilidades de resolución de problemas y de emisión de juicios. Esto da cuenta de que se aplicó la competencia de pensamiento crítico; competencia en la que el pensamiento ejecuta una serie de habilidades cognitivas y disposiciones con el fin de reflexionar la situación que se le presenta al individuo para darle solución o emitir un juicio.

El estudiantado de Nuevo León, de primer grado de secundaria, tiene orientación hacia el aprendizaje, investiga, reconoce sus capacidades, comparte su conocimiento, tiene apertura mental, pone atención, es organizado. Se siente motivado hacia la superación personal, demostrar que puede hacer las cosas, obtener buenas calificaciones, llegar a ser profesionales y mejorar económicamente. Estas características le facilitan el poner en práctica su competencia de pensamiento crítico y aprender mejor, ya que se aprecia la disposición de ir más allá de lo simple y existe la motivación por mejorar y superarse.

Las habilidades y disposiciones enunciadas son muy afines con lo que se expone en la teoría, pero faltó identificar muchas más habilidades del pensamiento, como la autorregulación, la meta-cognición, elaboración de preguntas y evaluar el razonamiento. Posiblemente, las habilidades antes mencionadas son más difíciles de alcanzar por estudiantes de secundaria, puesto que requieren mayor evaluación de sus pensamientos y razonamientos.

Un hallazgo muy valioso para la práctica educativa es conocer que los alumnos y alumnas competentes en pensamiento crítico muestran solidaridad, les gusta compartir su conocimiento y ayudar a quien se los solicita. Esto se puede tomar en cuenta para manejar la tutoría entre pares y elevar el aprendizaje del alumnado.

Sobresale el hallazgo de que es mínima la diferencia de competencia en desarrollo del pensamiento crítico entre hombres y mujeres, aunque en el contexto de aula se refleje más las capacidades de las mujeres y no las de los muchachos.

\section{Recomendaciones para futuros estudios}

Dados los resultados del presente estudio, sería conveniente una investigación más amplia en cuestión del tamaño de la muestra y de la población. Esto favorecería la ratificación y ampliación de los resultados aquí obtenidos para poder llegar más acertadamente a una generalización.

También es recomendable realizar un estudio sobre cómo se da la meta-cognición en el estudiantado de secundaria, o por qué no se da este proceso en este nivel educativo. Con la información que se pueda obtener se tendrían elementos para impulsar la comprensión y un nivel más alto de pensamiento crítico, a fin de contribuir a una mejor educación. 
doi: http://dx.doi.org/10.15359/ree.20-1.11

URL: http://www.una.ac.cr/educare

CORREO: educare@una.cr

Otra investigación podría girar sobre un comparativo de características de estudiantes por grado o nivel educativo; de esa forma se apreciaría la evolución o el decremento de cualidades. Lo anterior generaría un proceso más que sería encontrar las causas de tales movimientos y soluciones o acciones para favorecer las prácticas educativas en función de elevar la calidad de la educación.

Estas recomendaciones están planteadas para el contexto educativo de escuelas secundarias de México. Sin embargo, por ser la competencia desarrollo de pensamiento crítico una competencia genérica que impacta en varias áreas del conocimiento, los resultados de estas investigaciones pueden ser valorados en otros contextos como referencia para tomar conocimientos que pueden integrar a los propios, para realizar investigaciones propias y para hacer estudios comparativos, entre otras cosas.

\section{Referencias}

Bailin, S. (1999). The problem with Percy: Epistemology, understanding and critical thinking [EI problema de Percy: Epistemología, comprensión y pensamiento crítico]. Informal Logic, 19(2-3), 161-170. Recuperado de http://ojs.uwindsor.ca/ojs/leddy/index.php/informal logic/article/view/2325/1768

Beltrán, M. J. y Torres, N. Y. (Diciembre, 2009). Caracterización de habilidades de pensamiento crítico en estudiantes de educación media a través del test HCTAES. Zona Próxima, 11, 6685. Recuperado de http://www.redalyc.org/articulo.oa?id=85313003005

Cruz, A. (2010). Competencia para el desarrollo del pensamiento crítico con énfasis en habilidades cognitivas [objeto de aprendizaje]. Recuperado de http://www.ruv.itesm.mx/convenio/ catedra/oas/dpcehc/homedoc.htm

Delors, J. (1997). La educación encierra un tesoro. Informe a la Unesco de la Comisión Internacional sobre la educación para el siglo XXI. México: Santillana.

Dewey, J. (1998). Cómo pensamos. Nueva exposición de la relación entre el pensamiento reflexivo y proceso educativo. Barcelona: Paidós.

Díaz-Barriga, F. (Setiembre, 2001). Habilidades de pensamiento crítico sobre contenidos históricos en alumnos de bachillerato. Revista Mexicana de Investigación Educativa, 6(13), 525-554. Recuperado de http://www.redalyc.org/articulo.oa?id=14001308

Duran, M. y Şendag, S. (Abril, 2012). A preliminary investigation into critical thinking skills of urban high school students: role of an IT/STEM program [Una investigación preliminar sobre las habilidades de pensamiento crítico de los estudiantes de secundaria urbana: El papel del programa IT/STEM]. Creative Education, 3(2), 241-250. doi: http://dx.doi. org/10.4236/ce.2012.32038 
Ennis, R. H. (1993, verano). Critical thinking assessment [Evaluación del pensamiento crítico]. Theory Intro Practice, 32(3), 179-186. Recuperado de http://www3.qcc.cuny.edu/WikiFiles/ file/Ennis\%20Critical\%20Thinking\%20Assessment.pdf

Ennis, R. H. (1996). Critical thinking dispositions: their nature and assessibility [Disposiciones del pensamiento crítico: Su naturaleza y accesibilidad]. Informal Logic, 18(2-3), 165-182. Recuperado de http://ojs.uwindsor.ca/ojs/leddy/index.php/informal logic/article/ viewFile/2378/1820

Escurra, M. y Delgado, A. (2008). Relación entre disposición hacia el pensamiento crítico y estilos de pensamiento en alumnos universitarios de Lima metropolitana. Persona, 11, 143-175. Recuperado de http://www.redalyc.org/articulo.oa?id=147117608009

Facione, P. A. (1990). Critical thinking: A statement of expert consensus for purposes of educational assessment and instruction [Pensamiento Crítico: Una declaración consensuada de expertos con propósitos de evaluación educacional e instrucción]. California: The California Academic Press.

Facione, P. A. (2007). Pensamiento crítico: ¿Qué es y por qué es importante? Estados Unidos: Insight Assessment. Recuperado de http://www.eduteka.org/PensamientoCriticoFacione.php

García-San Pedro, M. J. (2009). El concepto de competencias y su adopción en el contexto universitario. Revista Alternativas. Cuadernos de Trabajo Social, 16, 11-28. Recuperado de http://rua.ua.es/dspace/bitstream/10045/13540/1/ALT 16 01.pdf

Giancarlo, C. A., Blohm, S. W. y Urdan, T. (Abril, 2004). Assessing secondary students' disposition toward critical thinking: Development of the California measure of mental motivation [La evaluación de la disposición de estudiantes de secundaria hacia el pensamiento crítico: Desarrollo del test California de la motivación mental]. Educational and Psychological Measurement, 64(2), 347-364. doi: http://dx.doi.org/10.1177/0013164403258464

Glaser, R. (1985). Thoughts on expertise. Learning research and development center University of Pittsburgh (Technical Report 8) [Reflexiones sobre la experiencia. Investigación, aprendizaje y centro de desarrollo de la Universidad de Pittsburgh (Reporte técnico 8)]. Recuperado de: http://www.dtic.mil/cgi-bin/GetTRDoc?AD=ADA157394

Guzmán, S. y Sánchez, P. (2006). Efectos de un programa de capacitación de profesores en el desarrollo de habilidades de pensamiento crítico en estudiantes universitarios en el sureste de México. REDIE. Revista Electrónica de Investigación Educativa, 8(2), 1-17. Recuperado de http://www.redalyc.org/articulo.oa?id=15508202 
doi: http://dx.doi.org/10.15359/ree.20-1.11

URL: http://www.una.ac.cr/educare

CORREO: educare@una.cr

Halpern, D. (1998). Teaching critical thinking for transfer across domains [La enseñanza del pensamiento crítico para la transferencia a través de dominios]. American Psychologist Associations, 53(4), 449-455. doi: http://dx.doi.org/10.1037/0003-066X.53.4.449

Hernández, R., Fernández, C. y Baptista, P. (2010). Metodología de la investigación. México: McGraw-Hill.

Kuhn, D. (Marzo, 1999). A developmental model of critical thinking [Un modelo de desarrollo del pensamiento crítico]. Educational Researcher, 28(2), 16-46. doi: http://dx.doi. org/10.3102/0013189X028002016

Lipman, M. (Septiembre, 1988). Critical thinking - what can it be? [Pensamiento crítico - ¿lo que puede ser?]. Educational Leadership, 46(1), 38-43. Recuperado de http://www.ascd.org/ ASCD/pdf/journals/ed lead/el 198809 lipman.pdf

López, G. (Enero-diciembre, 2012). Pensamiento crítico en el aula. Docencia e Investigación, 37(22), 41-60. Recuperado de http://educacion.to.uclm.es/pdf/revistaDI/3 22 2012.pdf

Madariaga, P. y Schaffernicht, M. (Julio-setiembre, 2013). Uso de objetos de aprendizaje para el desarrollo del pensamiento crítico. Revista de Ciencias Sociales, 19(3), 472-484. Recuperado de http://www.redalyc.org/pdf/280/28028572010.pdf

McShane, P., Gillis, A. y Benton, J. (2013). Introducción al pensamiento crítico. Madrid: Plaza y Valdés.

Montoya, J.I. (Mayo-agosto, 2007).Primer avance de investigación. Acercamiento al desarrollo del pensamiento crítico, un reto para la educación actual. Revista Virtual Universidad Católica del Norte, 21, 1-17. Recuperado de http://www.redalyc.org/articulo.oa?id=194220390001

Montoya, J. I. y Monsalve, J. C. (Septiembre-diciembre, 2008). Estrategias didácticas para fomentar el pensamiento crítico en el aula. Revista Virtual Universidad Católica del Norte, 25, 1-26. Recuperado de http://www.redalyc.org/articulo.oa?id=194215513012

Nieto, A. M. y Saiz, C. (2011). Skills and dispositions of critical thinking: Are they sufficient? [Habilidades y disposiciones sobre el pensamiento crítico: ¿Son suficientes?]. Anales de Psicología, 27(1), 202-209. Recuperado de http://revistas.um.es/analesps/article/ view/113641/107631

Olivares, S. L. y Heredia, Y. (2012). Desarrollo del pensamiento crítico en ambientes de aprendizaje basado en problemas en estudiantes de educación superior. Revista Mexicana de Investigación Educativa, 17(54), 759-778. Recuperado de http://www.scielo.org.mx/pdf/ rmie/v17n54/v17n54a4.pdf 
doi: http://dx.doi.org/10.15359/ree.20-1.11

URL: http://www.una.ac.cr/educare

CORREO: educare@una.cr

Organización para la Cooperación y el Desarrollo Económico (OCDE, 2005). La definición y selección de competencias clave (Resumen ejecutivo). Recuperado de http://www.deseco. admin.ch/bfs/deseco/en/index/03/02.parsys.78532.downloadList.94248.DownloadFile .tmp/2005.dscexecutivesummary.sp.pdf

Organización para la Cooperación y el Desarrollo Económico (OCDE, 2010). Habilidades y competencias del siglo XXI para los aprendices del nuevo milenio en los países de la OCDE. París: Instituto de Tecnologías Educativas. Recuperado de http://recursostic.educacion. es/blogs/europa/media/blogs/europa/informes/Habilidades y competencias siglo21 OCDE.pdf

Paul, R. y Elder, L. (2003). La mini-guía para el pensamiento crítico, conceptos y herramientas. California: Fundación para el Pensamiento Crítico. Recuperado de https://www. criticalthinking.org/resources/PDF/SP-ConceptsandTools.pdf

Paul, R. y Elder, L. (2005). Una guía para los educadores en los estándares de competencia para el pensamiento crítico. California: Fundación para el Pensamiento Crítico.

Perrenoud, P. (2008). Construir competencias, ¿es darle la espalda a los saberes? Revista de Docencia Universitaria, 2, 1-8. Recuperado de http://www.urosario.edu.co/CGTIC/ Documentos/construir competencias saberes.pdf

Quecedo, R. y Castaño, C. (2002). Introducción a la metodología de la investigación cualitativa. Revista Psicodidáctica, 14, 1-27. Recuperado de http://www.redalyc.org/articulo. oa?id=17501402

Real Academia Española. (2001). Diccionario de la lengua española (22 ${ }^{\mathrm{a}}$ ed.). Recuperado de http://lema.rae.es/drae/?val=pensamiento

Ruiz, S. D. (Junio-diciembre, 2013). Working by projects: A way to enrich critical thinking and the writing process in a third grade eflclassroom [Trabajar por proyectos: Una manera de enriquecer el pensamiento crítico y el proceso de escritura en una clase de tercer grado]. Colombian Applied Linguistics Journal, 15(2), 205-220. doi: http://dx.doi.org/10.14483/ udistrital.jour.calj.2013.2.a04

Secretaría de Educación Pública (SEP). (2011). Plan de estudios 2011. Educación básica. México: Autor.

Sierra, J., Carpintero, E. y Pérez, L. (2010). Pensamiento crítico y capacidad intelectual. Faísca, 15(17), 98-110. Recuperado de http://sid.usal.es/idocs/F8/ART21098/sierra paz.pdf

Sobrado, L. y Fernández, E. (2010). Competencias emprendedoras y desarrollo del espíritu empresarial en los centros educativos. Educación XXI, 13(1), 15-38. Recuperado de http:// www.redalyc.org/articulo.oa?id=70618037001 
doi: http://dx.doi.org/10.15359/ree.20-1.11

URL: http://www.una.ac.cr/educare

CORREO: educare@una.cr

Thomas, I. (2009). Critical thinking, transformative learning, sustainable education, and problembased learning in universities [El pensamiento crítico, el aprendizaje transformativo, la educación sostenible y el aprendizaje basado en problemas en las universidades].Journal of TransformativeEducation, 7(3), 245-264. doi: http://dx.doi.org/10.1177/1541344610385753

Tishman, S., Jay, E. y Perkins, D. N. (1993). Teaching thinking dispositions: From transmission to enculturation [Enseñando disposiciones de pensamiento: De la transmisión a la enculturación]. Theory Info Practice, 32(3), 144-153. doi: http://dx.doi. org/10.1080/00405849309543590

Unesco. (2005). Hacia las sociedades del conocimiento. París: Autor. Recuperado de http:// unesdoc.unesco.org/images/0014/001419/141908s.pdf

Valenzuela, J. R. y Flores, M. (2012). Fundamentos de investigación educativa. Nuevo León, México: Editorial Digital Tecnológico de Monterrey.

\section{(9) Cómo citar este artículo en APA:}

Molina-Patlán, C., Morales-Martínez, G. P. y Valenzuela-González, J. R. (Enero-abril, 2016). Competencia transversal pensamiento crítico: Su caracterización en estudiantes de una secundaria de México. Revista Electrónica Educare, 20(1), 1-26. doi: http://dx.doi.org/10.15359/ree.20-1.11

Nota: Para citar este artículo en otros sistemas puede consultar el hipervínculo "Como citar el artículo" en la barra derecha de nuestro sitio web: http://www.revistas.una.ac.cr/index.php/EDUCARE/index 